\title{
The influence of Unruh effect on quantum steering for accelerated two-level detectors with different measurements
}

\author{
Tonghua Liu ${ }^{1}$, Jieci Wang ${ }^{1,2 *}$, Jiliang Jing ${ }^{1}$, and Heng Fan ${ }^{2}$ \\ 1 Department of Physics, and Synergetic Innovation Center for Quantum Effects \\ and Applications, Hunan Normal University, Changsha, Hunan 410081, China \\ 2 Institute of Physics, Chinese Academy of Sciences, Beijing 100190, China
}

\begin{abstract}
We study the dynamics of steering between two correlated Unruh-Dewitt detectors when one of them locally interacts with external scalar field via different quantifiers. We find that the quantum steering, either measured by the entropic steering inequality or the Cavalcanti-Jones-Wiseman-Reid inequality, is fragile under the influence of Unruh thermal noise. The quantum steering is found always asymmetric and the asymmetry is extremely sensitive to the initial state parameter. In addition, the steering-type quantum correlations experience "sudden death" for some accelerations, which are quite different from the behaviors of other quantum correlations in the same system. It is worth noting that the domination value of the tight quantum steering exists a transformation point with increasing acceleration. We also find that the robustness of quantum steerability under the Unruh thermal noise can be realized by choosing the smallest energy gap in the detectors.
\end{abstract}

PACS numbers: 04.62.+v, 03.70.+k, 06.20.-f, 05.30.Jp

\footnotetext{
*Email: jieciwang@iphy.ac.cn
} 


\section{INTRODUCTION.}

Quantum steering, an intermediate quantum correlation between entanglement [1] and Bell nonlocality [2], was proposed by Schrödinger in 1935 [3, 4]. Steering is a phenomenon that allows one party, Alice, to steer the state of a distant party Bob by exploiting the shared entanglement. This fascinating phenomenon is regarded at the heart of the EinsteinPodolksy-Rosen [5] paradox and as a characteristic trait of quantum mechanics. Quantum steering has been intensively investigated in most recent years [6-12] after the realization that, apart from its foundational importance in both fundamental and practical perspective of quantum information, it is advantageous to perform some quantum information processing tasks [12, 13]. Despite many efforts on understanding the quantum steering [14-22], unlike entanglement and nonlocality in which a variety of measures exist, there is still scarce literature concerning the quantification of the quantum steerability [23-25].

On the other hand, a famous achievement in quantum field theory is the Unruh effect [26, 27], which indicates that quantum properties of fields are observer dependent. The Unruh effect reveals the fact that an uniformly accelerated detector in the Minkowski vacuum will detect thermal radiation at a temperature related to the proper acceleration of the detector. While the mathematical derivation of the Unruh effect is well established, direct observation of the Unruh effect in laboratories is still lacking since an observable Unruh temperature requires currently experimental unreachable acceleration. To get closer to Unruh's original derivation, people have modeled the observer by a point like two-level atom, which is named Unruh-DeWitt detector [28]. The detector is semiclassical because it possesses a classical world line while its internal degree of freedom is treated quantum mechanically. In addition, the study of quantum information aspects of the Unruh effect is essentially related to the information loss problem [29, 30] of black holes because, according to the equivalent principle, an accelerated observer in the Minkowski vacuum corresponds to an observer who hovers outside the event horizon of a black hole [31 36$]$.

In this paper we propose a tight measure of steering to analyze the quantum steering in a relativistically consistent way. We also study different measures of quantum steering between a pair of Unruh-Dewitt detectors when one of them is accelerated. Here we employ the Unruh-Dewitt detector model [28] rather than global free models [24] to study the behavior of quantum correlations in a relativistic setting [37 40] because the latter suffers 
from the single-mode approximate problem and physically unfeasible detection of quantum correlations in the full spacetime [41]. We find that the quantum steering, either measured by the entropic steering inequality or the Cavalcanti-Jones-Wiseman-Reid nonlocality steering inequality, is destroyed under the influence of the Unruh thermal noise. In addition, the entropic steering inequality measuring steering is found always asymmetry for any acceleration.

The outline of the paper is as follows. In Sec. II, we introduce two types of measurements to quantify steering and define a tight measure of steering. In Sec. III, we discuss the quantum information description of the accelerated Unruh-Dewitt detector and the evolution of the prepared state under the Unruh thermal bath. In Sec. IV, we study the behaviors of quantum steering in the relativistic setting with different measurements. Conclusions are given in the last section.

\section{QUANTIFICATIONS OF QUANTUM STEERING}

\section{A. Quantum steering based on the entropic inequality}

Most recently, a quantification for quantum steering [24] was performed based on the entropic uncertainty relations [16]. For a bipartite system $\rho_{A B}$ with two observables $\hat{R}$ and $\hat{O}$ in the $N$-dimensional Hilbert space, the quantum steering can be obtained by performing measurements on either the subsystem $A$ ( $A$-measuring) or the subsystem $B$ ( $B$-measuring). We start with the entropic steering inequality for the $A$-measuring case, which has the form [16]

$$
H\left(R^{B} \mid R^{A}\right)+H\left(O^{B} \mid O^{A}\right) \geq-\log \left(\Omega^{B}\right),
$$

where $\Omega \equiv \max _{\{i, j\}}\left\{\left|\left\langle R_{i} \mid O_{j}\right\rangle\right|^{2}\right\}$, and $\Omega^{B}$ is the value $\Omega$ associated with observables $\hat{R}^{B}$ and $\hat{O}^{B} \cdot H(\cdot)$ is the von Neumann entropy, and $H\left(R^{B} \mid R^{A}\right)$ is the conditional entropy after measurements on the subsystem A. In this paper we employ the measurements in the Pauli $X, Y$ and $Z$ bases on each side and consider a complete sets of pairwise complementary (mutually unbiased) observables. Then one can obtain the following form of entropic steering inequality of $A \rightarrow B$

$$
S I^{A \rightarrow B}=H\left(\sigma_{x}^{B} \mid \sigma_{x}^{A}\right)+H\left(\sigma_{y}^{B} \mid \sigma_{y}^{A}\right)+H\left(\sigma_{z}^{B} \mid \sigma_{z}^{A}\right) \geq 2 .
$$


Basing on the entropic steering inequality, we obtain the steering of the $A$-measuring case to quantify how much the bipartite state $\rho$ is steerable by Alice's measurements [24]

$$
S^{A B}:=\max \left\{0, \frac{S I^{A \rightarrow B}-2}{S I_{\max }-2}\right\},
$$

where $S I_{\max }$ is obtained when the entropic steering inequality is maximal violated for a given state and equals to 6 when the given state is a maximally entangled state [24]. Similarly, the steering for the $B$-measuring case can be defined by exchanging the roles of $A$ and $B$ :

$$
S^{B A}:=\max \left\{0, \frac{S I^{B \rightarrow A}-2}{S I_{\max }-2}\right\} .
$$

\section{B. Quantum steering based on the CJWR inequality}

Alternatively, Cavalcanti, Jones, Wiseman, and Reid (CJWR) developed [14] an inequality to judge whether a bipartite state is steerable. Later R. M. Angelo et.al propose a measure of steering basing on the maximal violation of the CJWR steering inequalities [25]. The CJWR inequality for a bipartite state $\rho$ has the form

$$
F_{n}(\rho, \mu)=\frac{1}{\sqrt{n}}\left|\sum_{i=1}^{n}\left\langle A_{i} \otimes B_{i}\right\rangle\right| \leqslant 1,
$$

where $F_{n}$ is a real-valued function and $A_{i}=\vec{u}_{i} \cdot \vec{\sigma}, B_{i}=\vec{v}_{i} \cdot \vec{\sigma}$ are two sets of observables of the system. In Eq. (5),$\vec{\sigma}=\left(\sigma_{1}, \sigma_{2}, \sigma_{3}\right)$ is the Pauli matrices, and $\vec{u}_{i} \in \mathbb{R}^{3}$ are unit vectors; $\vec{v}_{i} \in \mathbb{R}^{3}$ are orthonormal vectors, and $\mu=\left\{\vec{u}_{1}, \ldots, \vec{u}_{n}, \vec{v}_{1}, \ldots, \vec{v}_{n}\right\}$ is a set of measurement directions. Here we only consider the quantum system in which Alice and Rob are both allowed to measure two observables. More specifically, we assume that Alice and Rob each select anyone of two dichotomic measurements $\left\{A_{1}, A_{2}\right\},\left\{B_{1}, B_{2}\right\}$ for different directions and the outcomes of the observable $A$ are labeled $a \in\{-1,+1\}$ and similarly for other measurements. For a two-observable quantum system, it has been proven in [25] that the quantum steering can be quantified by

$$
S_{2}:=\max \left\{0, \frac{F_{2}(\rho)-1}{F_{2}^{\max }-1}\right\}
$$

where $F_{2}(\rho)=\max _{\mu}\left[F_{2}(\rho, \mu)\right]$ is taken over all measurement settings $\mu$ and $F_{2}^{\max }=$ $\max _{\rho}\left[F_{2}(\rho)\right]$ is the maximal value takes over all the bipartite states

$$
F_{2}(\rho)=\sqrt{c^{2}-c_{\min }^{2}}
$$


In Eq. (77) $c=\sqrt{\vec{c}^{2}}, c_{\text {min }} \equiv \min \left\{\left|c_{1}\right|,\left|c_{2}\right|,\left|c_{3}\right|\right\}$, and $c_{i}$ are components of the bloch sphere vector $\vec{c}$ in bloch sphere expansion of the state $\rho$ [42]

$$
\rho=\frac{1}{4}\left(\mathbb{I} \otimes \mathbb{I}+\vec{a} \cdot \vec{\sigma} \otimes \mathbb{I}+\mathbb{I} \otimes \vec{b} \cdot \vec{\sigma}+\sum_{r=1}^{3} c_{i} \sigma_{i} \otimes \sigma_{i}\right),
$$

where $\mathbb{I}$ is the $2 \times 2$ unit matrix and $\{\vec{a}, \vec{b}, \vec{c}\} \in \mathbb{R}^{3}$ are vectors with norm length smaller

than $\mathbb{I}$. The value of $F_{2}^{\max }$ can be determined by using the inequality $\vec{a}^{2}+\vec{b}^{2}+\vec{c}^{2} \leqslant 3$ with $\vec{a}^{2}=\vec{b}^{2}=0$. Hence, $F_{2}^{\max }=\sqrt{2}[14]$.

\section{Tight measure of steering}

In the last two subsections we have introduced two types of measurements for quantum steering based on the entropic steering inequality and the CJWR inequality, respectively. However, it is worth noting that there may be different types of steering inequalities for a given quantum state and each type of them is not superior to all other forms of steering inequalities. For example, for some states the steering can be measured by the violation of the entropic steering inequality but can't be measured by the violation of the CJWR inequalities. In other words, the state may violate entropic steering inequality but fail to violate CJWR steering inequality, vice versa. In addition, the first measure fails to give united amount of quantum steerability for different measuring directions, which makes the quantum steering inherent asymmetric. On the other hand, the second measures can't distinguish the notions of steering and Bell nonlocality in the two-measurement scenario [25]. To obtain an united quantifier, we propose a more tight limit for the measure of steering based on the aforementioned quantifiers. That is, for a given state, it is steerable only if all the above mentioned steering inequalities are violated with the same set of measurements. Therefore, the tight quantum steerability of a bipartite system is defined by minimuming the violation over all the inequalities, which is

$$
\mathrm{S}_{g} \equiv \min \left\{S^{1}, S^{2}, \ldots S^{n}\right\}
$$

where $S^{1}, S^{2}, \ldots S^{n}$ are different quantifiers basing on the violation of different steering inequalities for the same state. Here we have $S^{1}, S^{2}, \ldots S^{n}=S^{A B}, S^{B A}, S_{2}$. 


\section{EVOLUTION OF THE DETECTORS' STATE UNDER RELATIVISTIC MO- TION}

In this section we give a brief description for the Unruh-Dewitt detectors [28] from a quantum information viewpoint and discuss the evolution of the system when one detector experiences relativistic motion. The Unruh-Dewitt detector is modeled by a two-level atom which interacts only with its nearby fields. We assume that the detectors are initially share some quantum correlations in the Minkowski spacetime and are observed by Alice and Bob, respectively. The atom carried by Alice keeps inertial and is always switched off while Rob's detector interacts with the scalar field when it moves with uniform acceleration for a time duration $\Delta$. The world line of Rob's detector is

$$
t(\tau)=a^{-1} \sinh a \tau, x(\tau)=a^{-1} \cosh a \tau,
$$

$y(\tau)=z(\tau)=0$, where $a$ is detector's proper acceleration. In Eq. (10) $\tau$ is proper time of the detector and $(t, x, y, z)$ are the usual Cartesian coordinates in the Minkowski spacetime. Throughout this paper, we employ natural units $c=\hbar=\kappa_{B}=1$. Since Rob's detector interacts with the field, the interaction should be taken into account and the total system evolves to a tripartite one. The initial state of the total system is

$$
\left|\Psi_{t_{0}}^{A R \phi}\right\rangle=\left|\Psi_{A R}\right\rangle \otimes\left|0_{M}\right\rangle
$$

where $\left|\Psi_{A R}\right\rangle=\sin \theta\left|0_{A}\right\rangle\left|1_{R}\right\rangle+\cos \theta\left|1_{A}\right\rangle\left|0_{R}\right\rangle$ denotes the initial state shared by Alice's (A) and Rob's $(\mathrm{R})$ detectors, and $\left|0_{M}\right\rangle$ represent that the external scalar field is in Minkowski vacuum.

The total Hamiltonian of the system is given by

$$
H_{A R \phi}=H_{A}+H_{R}+H_{K G}+H_{\mathrm{int}}^{R \phi},
$$

where $H_{A}=\Omega A^{\dagger} A$ and $H_{R}=\Omega R^{\dagger} R$ are Hamiltonians of the detectors and $H_{K G}$ is Hamiltonian of the external scalar field; $\Omega$ is the energy gap of the detectors. In Eq. (12) the

interaction Hamiltonian $H_{\text {int }}^{R \phi}(t)$, which describes how Rob's detector is coupled with the external massless scalar field $\phi(x)$, is given by

$$
H_{\mathrm{int}}^{R \phi}(t)=\epsilon(t) \int_{\Sigma_{t}} d^{3} \mathbf{x} \sqrt{-g} \phi(x)\left[\chi(\mathbf{x}) R+\bar{\chi}(\mathbf{x}) R^{\dagger}\right]
$$


where $g \equiv \operatorname{det}\left(g_{a b}\right), g_{a b}$ is the Minkowski metric, and $\mathbf{x}$ are coordinates defined on the Cauchy surface $\sum$ associated with the timelike isometries followed by the qubits. In Eq. (13), $\epsilon(t)$ is a real-valued switching function which keeps the detector switched on smoothly for a finite amount of proper time $\Delta$ and $\chi(\mathbf{x})=(\kappa \sqrt{2 \pi})^{-3} \exp \left(-\mathbf{x}^{2} / 2 \kappa^{2}\right)$ is a point-like coupling function which guarantees the detector is space-localized and only interacts with the field in a neighborhood of its world line. By combining the detector-field free Hamiltonian as $H_{0}=H_{A}+H_{R}+H_{K G}$, one can cast the total Hamiltonian as

$$
H_{R \phi}=H_{0}+H_{\text {int }}
$$

The state $\left|\Psi_{t}^{A R \phi}\right\rangle$ at time $t=t_{0}+\Delta$ can be written as

$$
\left|\Psi_{t}^{A R \phi}\right\rangle=T \exp \left[-i \int_{t_{0}}^{t} d t H_{\mathrm{int}}^{I}(t)\right]\left|\Psi_{t_{0}}^{A R \phi}\right\rangle
$$

in the interaction picture, where $T$ is the time-ordering operator. In Eq. (15), $H_{\text {int }}^{I}(t)=$ $U_{0}^{\dagger}(t) H_{\text {int }}(t) U_{0}(t)$ and $U_{0}(t)$ is the unitary evolution operator associated with the Hamiltonian $H_{0}(t)$. Then the final state $\left|\Psi_{t}^{A R \phi}\right\rangle$ is found to be

$$
\begin{aligned}
\left|\Psi_{t}^{A R \phi}\right\rangle & =T \exp \left[-i \epsilon(t) \int d^{4} x \sqrt{-g} U_{0}^{\dagger}(t) \phi(x)\left(\chi(\mathbf{x}) R+\bar{\chi}(\mathbf{x}) R^{\dagger}\right) U_{0}(t)\right]\left|\Psi_{t_{0}}^{A R \phi}\right\rangle \\
& =T \exp \left[-i \epsilon(t) \int d^{4} x \sqrt{-g} U_{0}^{\dagger}(t) \phi(x) U_{0}(t)\left(\chi(\mathbf{x}) e^{-i \Omega t} e^{i \Omega t} R+\bar{\chi}(\mathbf{x}) e^{i \Omega t} e^{-i \Omega t} R^{\dagger}\right)\right]\left|\Psi_{t_{0}}^{A R \phi}\right\rangle \\
& =T \exp \left[-i \int d^{4} x \sqrt{-g} \phi^{I}(x)\left(f R^{I}+\bar{f} R^{\dagger I}\right)\right]\left|\Psi_{t_{0}}^{A R \phi}\right\rangle,
\end{aligned}
$$

where $f \equiv \epsilon(t) e^{-i \Omega t} \chi(\mathbf{x})$ and $R^{I}=e^{i \Omega t} R$ have been used. The dynamics of the atom-field system at time $t=t_{0}+\Delta$ can be calculated in the first order of perturbation over the coupling constant $\epsilon$ [28]. Under the dynamic evolution described by the Hamiltonian given by Eq.(13), the final state of the system is [31, $37-40]$

$$
\left|\Psi_{t}^{A R \phi}\right\rangle=\left[I-i\left(\phi(f) R+\phi(f)^{\dagger} R^{\dagger}\right)\right]\left|\Psi_{t_{0}}^{A R \phi}\right\rangle
$$

where the operator

$$
\begin{aligned}
\phi(f) & \equiv \int d^{4} x \sqrt{-g} \chi(x) f \\
& =i\left[a_{R I}(\overline{u E \bar{f}})-a_{R I}^{\dagger}(u E f)\right],
\end{aligned}
$$


is the distribution function of the external scalar field. In Eq. (17), $E f$ is approximately a positive-frequency solution of the scalar field [31, 37-40]. Besides, the $u$ operator is the positive-frequency part of the solutions of K-G equation with respect to the timelike isometry, and

$$
E f=\int d^{4} x^{\prime} \sqrt{-g\left(x^{\prime}\right)}\left[G^{\mathrm{adv}}\left(x, x^{\prime}\right)-G^{\mathrm{ret}}\left(x, x^{\prime}\right)\right] f\left(x^{\prime}\right),
$$

where $G^{\text {adv }}$ and $G^{\text {ret }}$ are the advanced and retarded Green functions. The annihilation operator $a_{R I}(\bar{u})$ annihilates the Rindler vacuum for the single mode $u$, which is an positivefrequency solution of the Klein-Gordon equation in the Rindler metric [31, 37-40].

By composing the initial state Eq. (11) and Eq. (16), the final state of the total system can be expressed in terms of the Rindler operators $a_{R I}^{\dagger}$ and $a_{R I}$

$$
\begin{aligned}
\left|\Psi_{t}^{A R \phi}\right\rangle & =\left|\Psi_{t_{0}}^{A R \phi}\right\rangle+\sin \theta\left|0_{A}\right\rangle\left|0_{R}\right\rangle \otimes\left(a_{R I}^{\dagger}(\lambda)\left|0_{M}\right\rangle\right) \\
& +\cos \theta\left|1_{A}\right\rangle\left|1_{R}\right\rangle \otimes\left(a_{R I}(\bar{\lambda})\left|0_{M}\right\rangle\right),
\end{aligned}
$$

where $\lambda=-u E f$. In Eq. (19), the creation and annihilation operators $a_{R I}^{\dagger}(\lambda)$ and $a_{R I}(\overline{\lambda)}$ are defined in the Rindler region $I$, while $\left|0_{M}\right\rangle$ is the Minkowski vacuum. The relations between these two sets of operators are [37, 40]

$$
\begin{aligned}
& a_{R I}(\bar{\lambda})=\frac{a_{M}\left(\overline{F_{1 \Omega}}\right)+e^{-\pi \Omega / a} a_{M}^{\dagger}\left(F_{2 \Omega}\right)}{\left(1-e^{-2 \pi \Omega / a}\right)^{1 / 2}}, \\
& a_{R I}^{\dagger}(\lambda)=\frac{a_{M}^{\dagger}\left(F_{1 \Omega}\right)+e^{-\pi \Omega / a} a_{M}\left(\overline{F_{2 \Omega}}\right)}{\left(1-e^{-2 \pi \Omega / a}\right)^{1 / 2}},
\end{aligned}
$$

where $F_{1 \Omega}=\frac{\lambda+e^{-\pi \Omega / a} \lambda \circ w}{\left(1-e^{-2 \pi \Omega / a}\right)^{1 / 2}}$, and $F_{2 \Omega}=\frac{\overline{\lambda \circ w}+e^{-\pi \Omega / a} \bar{\lambda}}{\left(1-e^{-2 \pi \Omega / a}\right)^{1 / 2}}$. Here $w(t, x)=(-t,-x)$ is a wedge reflection isometry that reflects $\lambda$ defined in the Rindler region $I$ into $\lambda \circ w$ in the other region $I I$ [31, 37, 40].

We are interested in the evolution of the detectors' state after interacting with the field. Therefore, the part for the external field $\phi(f)$ should be traced out. Then we obtain

$$
\rho_{t}^{A R}=\left(\begin{array}{cccc}
\gamma & 0 & 0 & 0 \\
0 & 2 \alpha \sin ^{2} \theta & \alpha \sin 2 \theta & 0 \\
0 & \alpha \sin 2 \theta & 2 \alpha \cos ^{2} \theta & 0 \\
0 & 0 & 0 & \beta
\end{array}\right)
$$



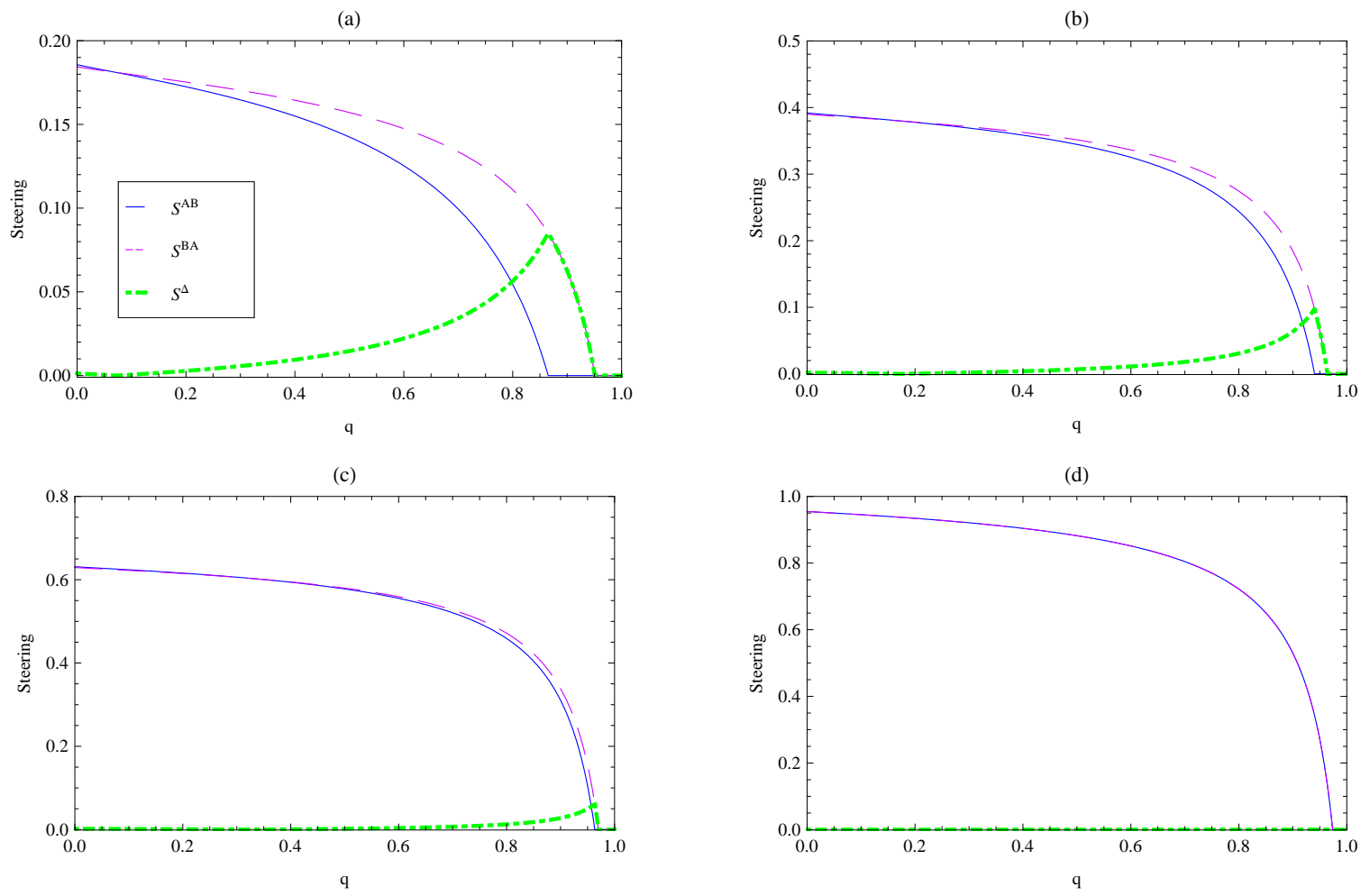

FIG. 1: Steering of $S^{A B}$ (blue solid line), $S^{B A}$ (purple dash line), steering asymmetry $S^{\Delta}$ (green dash line between the detectors as a function of acceleration parameter $q$. The initial state parameter $\theta$ and effective coupling parameter $\nu$ are: (a) $\nu=0.1, \theta=\pi / 12$, (b) $\nu=0.1, \theta=\pi / 8$, (c) $\nu=0.1, \theta=\pi / 6$, (d) $\nu=0.1, \theta=\pi / 4$, respectively.

for the detectors, where the parameters $\alpha, \beta$ and $\gamma$ have the following forms

$$
\begin{aligned}
& \alpha=\frac{1-q}{2(1-q)+2 \nu^{2}\left(\sin ^{2} \theta+q \cos ^{2} \theta\right)}, \\
& \beta=\frac{\nu^{2} q \cos ^{2} \theta}{(1-q)+\nu^{2}\left(\sin ^{2} \theta+q \cos ^{2} \theta\right)}, \\
& \gamma=\frac{\nu^{2} \sin ^{2} \theta}{(1-q)+\nu^{2}\left(\sin ^{2} \theta+q \cos ^{2} \theta\right)},
\end{aligned}
$$

respectively. In Eq. (22) the acceleration $a$ has been parametrized as $q \equiv e^{-2 \pi \Omega / a}$ and $\nu$ is a combined coupling parameter, which is $\nu^{2} \equiv\|\lambda\|^{2}=\frac{\epsilon^{2} \Omega \Delta}{2 \pi} e^{-\Omega^{2} \kappa^{2}}$ [37 40$]$. In our model $\epsilon \ll 1$ and $\nu^{2} \ll 1$ are required for the validity of the perturbation approach. 


\section{BEHAVIORS OF QUANTUM STEERING UNDER THE INFLUENCE OF UNRUH THERMAL NOISE}

In this section we study the behaviors of quantum steering under the influence of thermal field induced by the Unruh radiation. The initial state of the entire system is given in Eq. (11), from which we can see that the amount of the detectors' initial state $\left|\Psi_{A R}\right\rangle$ depends on $\theta$. For any nonzero $\theta$, the detector $A$ shares initial quantum correlation with detector $R$. Then Rob's detector is accelerated for a time duration $\Delta$ with constant acceleration and influenced by the Unruh thermal bath. We are interested in if the steering-type quantum correlations between the detectors is destroyed by the Unruh thermal noise and if the asymmetric of quantum steering from different directions is affected by the Unruh effect.

\section{A. Behaviors of quantum steering measured by the violation of the entropic in-} equality

We start with discussing the dynamics of quantum steerability measured by the violation of the entropic steering inequality under the influence of the Unruh radiation. We aware that the dynamics of entropic inequality measuring steering has been discussed for free bosonic

field modes [24]. However, the quantum correlations for free bosonic modes suffers from two disadvantages in a relativistic frame. The first one is the so called single-mode approximate problem [41], that is, one cannot map a single-frequency Minkowski mode with a singlefrequency Rindler mode. This is because an inertial observer in the Minkowski spacetime is freely to create excitations in any accessible modes in the accelerated frame [41]. Therefore, we have to choose the Minkowski modes as superpositions of different frequencies of Rindler modes. Secondly, the free field quantum correlation suffers from the unfeasible detection in the full spacetime because the spacetime is isolated by the Rindler event horizon. To overcome these disadvantages, here we employ the Unruh-Dewitt detector which interacts only with its nearby fields and locally detectable.

Since the violation of the entropic uncertainty relations measuring quantum steering depends on the direction of the performed measurements, it may be asymmetric [11] under different measuring parts. To better understand this property, we calculate the entropic steering $S^{A B}$ for the $A \rightarrow B$ direction and $S^{B A}$ for the $B \rightarrow A$ direction for the final state. 
To check the degree of steerability asymmetric, we also calculate the steering asymmetry, which is $S^{\Delta}=\left|S^{A B}-S^{B A}\right|$.

In Fig. (1) we plot the steering of $S^{A B}, S^{B A}$, as well as the steering asymmetry $S^{\Delta}$ versus the acceleration parameter $q$ for fixed coupling parameter $\nu=0.1$ and different initial state parameters. It is shown that both the quantum steering of $S^{A B}$ and $S^{B A}$ monotone degrade with the growth of the acceleration parameter $q$, which means that the thermal noise induced by Unruh radiation destroys the steering-type quantum resources. It is worth noting that the steering-type quantum correlations happen "sudden death" for some acceleration $q$, which is quite different form the behavior of discord-type correlation where the quantum discord has a "sudden change" point and approaches zero only in the limit of $q \rightarrow 1$ [38]. It is shown that the $B \rightarrow A$ steering is always bigger than the $A \rightarrow B$ with growing acceleration except the case of $\theta=\pi / 4$. Considering that they equal to each other when there are no acceleration $(q=0)$, we can conclude that the steering form the noninertial part to the inertial part is less affected by the Unruh thermal noise.

From Figs. (1a-1d) we can see that the steering asymmetry increases and then decreases with increasing acceleration parameter $q$. In addition, the maximal steering asymmetry appears when one of the two steering directions suffers from "sudden death". Furthermore, the "sudden death" point is in fact a critical point because only one direction is steerable after the one-way "sudden death". In other words, the system is currently experiencing a transformation under the influence of Unruh effect and the transformation will happen earlier for a smaller initial state parameter $\theta$. This result is nontrivial because it is shown that the asymmetry of steerability is extremely sensitive to the initial state parameter, which is a unique nature of quantum steering comparing with the behaviors of other forms of quantum correlations, such as quantum entanglement [37], quantum discord [38], quantum nonlocality [39] and quantum coherence [40] in the same system. We can see from Fig. (1d) that the steering of $S^{A B}$ is almost equivalent to $S^{B A}$, which means that the asymmetry of steering is very small when the initial state is a maximally entangled state.

We note that the effect of thermal noise on the quantum steering between two systems has previously been studied in [43-45]. For example, the authors examined the effect of an initial thermal excitation of an oscillator on observing an EPR paradox between an oscillator and a pulse in Ref. [43]. They found that the steering suffers "sudden death" for above a certain threshold value for thermal noise. Here we find that, similar with results for Gaussian 
states [43, 44], the thermal noise induced by the Unruh effect damages the steering and the "sudden death" also found for steering-type correlation. However, the source of thermal noise is attribute to thermal occupation of the mechanical oscillator, but the generation of thermal noise is come from the atoms acceleration of the detectors in our present paper. We also find that in the relativistic case the maximal steering asymmetry appears when one of the two steering directions suffers from "sudden death". In addition, the thermal bath induced by the Unruh radiation always generates asymmetry of steerability. These behaviors are not found in earlier papers in inertial systems.

\section{B. Behaviors of CJWR inequality measure of quantum steering and the tight measure of steering}

Now we have discussed the dynamics of entropic inequality measuring quantum steering between an inertial detector and an accelerated Unruh-Dewitt detector. However, as we stated before, this measure of steering fails to give united amount of quantum steerability for different measuring directions. In this subsection we calculate the CJWR inequality measure of quantum steering and the tight measure of steering for the final state Eq. (22) after the accelerated motion of Rob's detector. To this end we rewrite the final state Eq. (22) to a Bloch sphere expansion form with vectors $\vec{a}=\left(a_{1}, a_{2}, a_{3}\right), \vec{b}=\left(b_{1}, b_{2}, b_{3}\right), \vec{c}=\left(c_{1}, c_{2}, c_{3}\right)$. After some calculations the final state is given by a general form of bloch sphere expansion

$$
\rho_{t}^{A R}=\frac{1}{4}\left(\begin{array}{cccc}
a_{3}+b_{3}+c_{3}+1 & b_{1}-i b_{2} & a_{1}-i a_{2} & c_{1}-c_{2} \\
b_{1}+i b_{2} & a_{3}-b_{3}-c_{3}+1 & c_{1}+c_{2} & a_{1}-i a_{2} \\
a_{1}+i a_{2} & c_{1}+c_{2} & -a_{3}+b_{3}-c_{3}+1 & b_{1}+i b_{2} \\
c_{1}-c_{2} & a_{1}+i a_{2} & b_{1}+i b_{2} & -a_{3}-b_{3}+c_{3}+1
\end{array}\right),
$$

where

$$
\begin{aligned}
& a_{1}=a_{2}=b_{1}=b_{2}=0, \\
& c_{1}=c_{2}=2 \alpha \sin 2 \theta, \\
& a_{3}=4 \alpha \sin ^{2} \theta+2 \gamma-1, \\
& b_{3}=4 \alpha \cos ^{2} \theta+2 \gamma-1, \\
& c_{3}=1-4 \alpha .
\end{aligned}
$$


Using Eqs. (66) and (77) we obtain the CJWR inequality measure of quantum steering $S_{2}$ and plot them in Fig. (2).
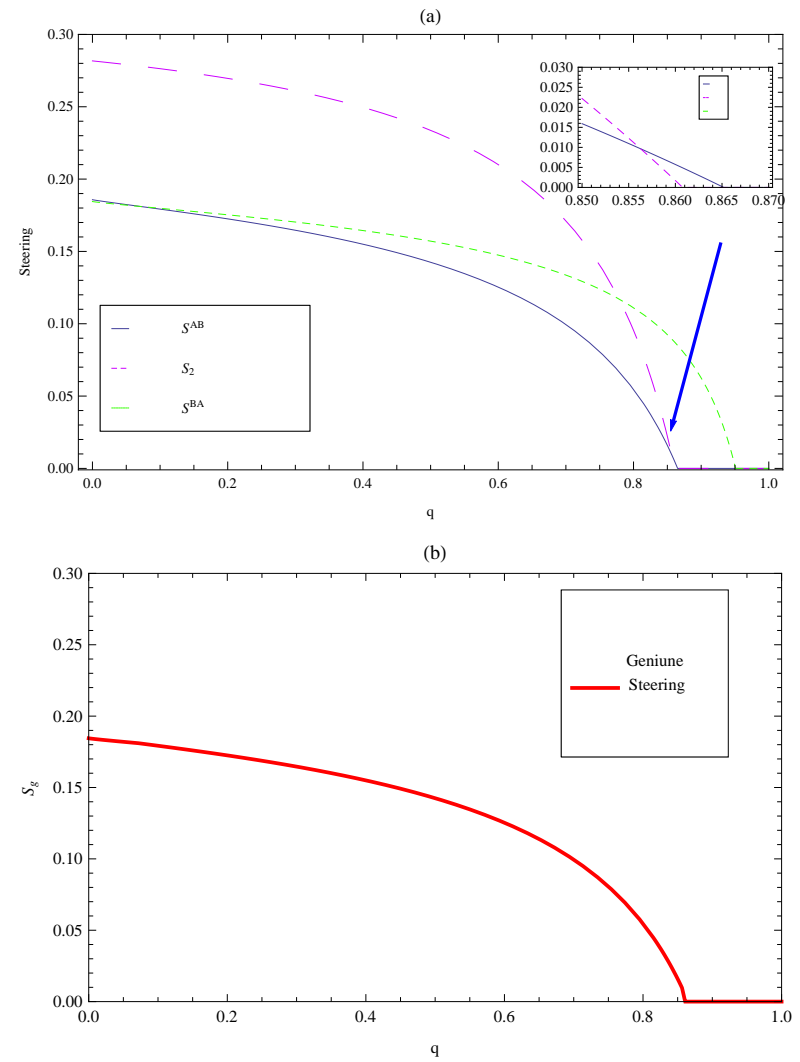

FIG. 2: (a) The quantum steering of $S_{2}, S^{A B}$, and $S^{B A}$ as a function of the acceleration parameter q. (b) The tight steering $\mathrm{S}_{g}$ between Alice and Rob as a function of the detector acceleration parameter $q$. The initial state parameter $\theta=\pi / 12$ and effective coupling parameter $\nu=0.1$

In Fig. (2a) we plot the behavior of the $S_{2}$ steering and compare it with the $S^{A B}$ and $S^{B A}$ steering. All the three measurements are plotted as a function of the acceleration parameter $q$ for fixed coupling parameter $\nu=0.1$ and initial state parameter $\theta=\pi / 12$. The variation trend of the curve of steering $S_{2}$ is similar with the entropic steering $S^{A B}$ and $S^{B A}$ and it also experiences "sudden death" for some accelerations. We find that both of the measures of steering monotone decrease with the increase of the acceleration parameter $q$. Here we have employed two types of quantifier for the steering of the final state and obtained similar results. It is also found that the CJWR inequality measure of quantum steering can only measure the degree of a bipartite steerable state but can't specific show the asysmmetic of quantum steering. This is because the steering inequality of the CJWR measure is equivalent 
with the inequality of Bell nonlocality where the system allows to measure two observables, which makes the notions of steering and Bell nonlocality derived here indistinguishable in the two-measurement scenario.

Now let us give some physical interpretations for the loss of quantum steering in the accelerated system. Alice performs trusted quantum measurements on her own subsystem, and Rob trusts his measurement apparatus. At this moment Alice is able to convince Rob (who does not trust Alice) because they share more quantum correlations initially. However, Alice gradually loses this ability when Rob's acceleration is growing because the shared quantum correlations are decreased under the Unruh thermal noise.

It is worth noting that the steering $S_{2}$ is more than the steering $S^{A B}$ and $S^{B A}$ for some small accelerations, but it deceases much more rapid than the latter two and appears an earlier "sudden death" with increasing acceleration. That is to say, there is no domination relation between these two types of steering quantifiers. Considering that the tight measure of steering $S_{g}$ is defined by minimuming the violation over all the inequalities, as has been shown in Eq. (9), $\mathrm{S}_{g}$ should take different parts from all the three steering measurements $S_{2}, S^{A B}$ and $S^{B A}$ for different accelerations.

In Fig. (2-b), we plot the tight measure of steering $S_{g}$ under the Unruh thermal noise as a function of the acceleration parameter $q$ for fixed coupling parameter $\nu=0.1$. We can see that the value of the tight quantum steering is dominated by the $S^{A B}$ steering for small acceleration, but the $S_{2}$ steering predominates the tight quantum steering when the acceleration parameter $q>0.857$. That is to say, $q=0.857$ is a critical point for the measure of tight quantum steering. We find again the tight quantum steering decreases with increasing acceleration of Rob's detector and appears "sudden death" . Then we arrive at the conclusion that the steering-type of quantum correlation is destroyed by the thermal bath induced by the Unruh effect.

We are also interested in how the detail of the interaction between the accelerated detector and external scalar field influences the steering-type quantum correlation. To this end we plot the tight steering $S_{g}$ of the system as functions of the energy gap $\Omega$ of Rob's detector and the interaction time $\Delta$ in Fig. (3). For the validity of the perturbation approach applied in this work, the parameters related to the effective coupling parameter $\nu$ are fixed to satisfy $\epsilon \ll \Omega^{-1} \ll \Delta$. It is shown in Fig. (3) that the steering-type quantum correlation is sensitive to the variation of different energy gaps of the accelerated 


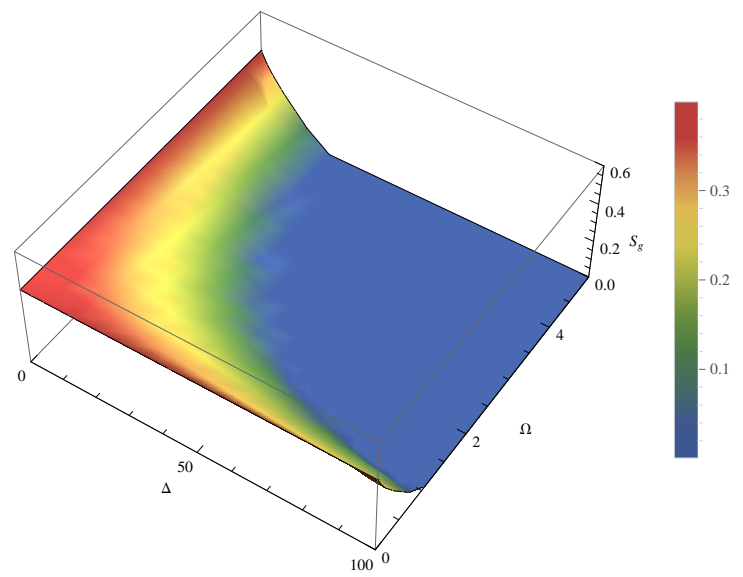

FIG. 3: The tight steering $\mathrm{S}_{g}$ between Alice and Rob as functions of the interaction time duration $\Delta$ and the energy gap $\Omega$. The parameters related to the effective coupling parameter $\nu$ are fixed as $\epsilon=2 \pi \cdot 10^{-4}$ and $\kappa=0.02$, respectively. The initial state parameter is $\theta=\pi / 8$ and the acceleration parameter is $q=0.5$.

detector. In particular, the quantum steering is much more robust over the interaction time when the energy gaps are small. On the other hand, the tight steering is very fragile under the interaction between the detector and the external field. The energy gap of the accelerated two-level atom has significant impact on the available quantum resource of the system. Therefore, we can prepare proper detectors via some artificial two-level atoms that possess proper energy gaps to obtain robust steering-type quantum correlation over the Unruh thermal noise. Alternatively, larger values of steering are obtained for a shorter interaction time.

\section{CONCLUSIONS}

In conclusion, we have studied the evolution of steering for two entangled Unruh-Dewitt detectors when one of them is accelerated and interacts with the neighbor external scalar field. We employ two different measures of steering based on the violation of the entropic steering inequality and the CJWR steering inequality, respectively. Then we define the tight steering basing on the minimal violation over all the inequalities. We find that the Unruh thermal noise will destroy the steering-type quantum resource for all the steering measurements. For the entropic inequality measure of quantum steering, it exists asym- 
metric property and the asymmetry of steerability is extremely sensitive to the initial state parameter $\theta$. It worth noting that the steering-type quantum correlations happen "sudden death", which is quite different form the behavior of the discord-type correlation in the limit of $q \rightarrow 1$. We find that the CJWR inequality measuring steering fails to specific how the asysmmetic of quantum steering and is indistinguishable from the Bell nonlocality. In addition, the tight measure of steering $S_{g}$ should take different parts from all the three steering measurements $S_{2}, S^{A B}$ and $S^{B A}$ for different accelerations. The domination value of the tight quantum steering is found to have a transformation point $q=0.857$ for the measurements of steering-type quantum correlation. It is also shown that robust tight steering under the Unruh thermal noise can be obtained by choosing the shortest interaction time allowed in quantum mechanics and some small energy gaps. We know that an accelerated observer in the Minkowski vacuum corresponds to a static observers outside a black hole

in the Hartle-Hawking vacuum [28, 32, 38]. Similarly, a static observer in the Minkowski space-time corresponds to a free-falling observer in the Schwarzschild spacetime. Therefore, the analysis used to derive the results of our manuscript can, in principle, be applied to study the dynamic behavior of quantum steering under the influence of Hawking radiation.

\section{Acknowledgments}

This work is supported by the National Natural Science Foundation of China under Grant No. 11675052, No. 11475061, and No. 91536108, the Doctoral Scientific Fund Project of the Ministry of Education of China under Grant No. 20134306120003, and the Postdoctoral Science Foundation of China under Grant No. 2014M560129, No. 2015 T80146.

[1] R.Horodecki,P.Horodecki,M.Horodecki,and K.Horodecki, Rev.Mod. Phys. 81, 865, (2009).

[2] N.Brunner,D.Cavalcanti,S.Pironio,V.Scarani,and S.Wehner, Rev.Mod. Phys. 86, 419, (2014).

[3] E. Schrodinger,Proc.Camb. Phil. Soc. 31, 553, (1935).

[4] E. Schrodinger, Proc. Camb. Phil. Soc. 32, 446 (1936).

[5] A. Einstein, B. Podolsky, and N. Rosen, Phys. Rev. 47, 777 (1935).

[6] V. Handchen et al., Nat. Photon. 6, 596 (2012); T. Guerreiro et al., Phys. Rev. Lett. 117, 070404 (2016). 
[7] S. Wollmann et al., Phys. Rev. Lett. 116, 160403 (2016); K. Sun et al., Phys. Rev. Lett. 116, $160404(2016)$.

[8] T. Moroder, O. Gittsovich, M. Huber, R. Uola, and O. Guhne Phys. Rev. Lett. 116, 090403 (2016); H. Zhu, M. Hayashi, and L. Chen Phys. Rev. Lett. 116, 070403 (2016); S. Chen, N. Lambert, C. Li, A. Miranowicz, Y.Chen, and F. Nori Phys. Rev. Lett. 116, 020503 (2016).

[9] Q. Y. He, Q. H. Gong, and M. D. Reid, Phys. Rev. Lett. 114, 060402 (2015).

[10] J. Bowles, T. Vértesi, M. T. Quintino, and N. Brunner, Phys. Rev. Lett. 112, 200402 (2014).

[11] I. Kogias, A. R. Lee, S. Ragy, and G. Adesso, Phys. Rev. Lett. 114, 060403 (2015).

[12] H. M.Wiseman, S. J. Jones, and A. C. Doherty, Phys. Rev. Lett. 98, 140402 (2007).

[13] C. Branciard and N. Gisin, Phys. Rev. Lett. 107, 020401 (2011).

[14] E. G. Cavalcanti, S. J. Jones, H. M. Wiseman, and M. D. Reid, Phys. Rev. A 80, 032112 (2009).

[15] S. P. Walborn, A. Salles, R. M. Gomes, F. Toscano, and P. H. Souto Ribeiro, Phys. Rev. Lett. 106, $130402(2011)$.

[16] J. Schneeloch, C. J. Broadbent, S. P. Walborn, E. G. Cavalcanti, and J. C. Howell, Phys. Rev. A 87, $062103(2013)$.

[17] E. C. G. Cavalcanti, M. J. W. Hall, and H. M. Wiseman, Phys. Rev. A 87, 032306 (2013).

[18] T. Pramanik, M. Kaplan, and A. S. Majumdar, Phys. Rev. A 90, 050305(R) (2014).

[19] S. L. W. Midgley, A. J. Ferris, and M. K. Olsen, Phys. Rev. A 81, 022101 (2010).

[20] Q. Y. He and M. D. Reid, Phys. Rev. Lett. 111, 250403 (2013).

[21] M. D. Reid, Phys. Rev. A 88, 062108 (2013).

[22] M. B. Plenio and S. Virmani, Quantum Inf. Comput. 7, 1 (2007).

[23] P. Skrzypczyk, M. Navascues, and D. Cavalcanti, Phys. Rev. Lett. 112, 180404 (2014).

[24] W.-Y. Sun, D. Wang and L. Ye, Laser Phys. Lett. 14, 095205 (2017).

[25] A. C. S. Costa and R. M. Angelo, Phys. Rev. A 93, 020103 (2016).

[26] W. G. Unruh, Phys. Rev. D 14, 870, (1976).

[27] L. Crispino et al., Rev. Mod. Phys. 80, 787 (2008).

[28] W. G. Unruh and R. M. Wald, Phys. Rev. D 29, 1047 (1984).

[29] L. Bombelli, R. K. Koul, J. Lee, and R. D. Sorkin, Phys. Rev. D 34, 373 (1986).

[30] S. W. Hawking, Commun. Math. Phys. 43, 199 (1975); Phys. Rev. D 14, 2460 (1976); H. Terashima, Phys. Rev. D 61, 104016 (2000). 
[31] R. M. Wald, Quantum Field Theory in Curved Spacetimes and Black Hole Thermodynamics (The University of Chicago Press, Chicago, 1994).

[32] I. Fuentes-Schuller and R. B. Mann, Phys. Rev. Lett. 95, 120404 (2005).

[33] T. G. Downes, I. Fuentes, and T. C. Ralph, Phys. Rev. Lett. 106, 210502 (2011); B. L. Hu, Shih-Yuin Lin, Jorma Louko, Class. Quantum Grav. 29, 224005 (2012).

[34] M. Ahmadi, D. E. Bruschi, and I. Fuentes, Phys. Rev. D 89, 065028 (2014).

[35] J. Wang, J. Jing, and H. Fan, Phys. Rev. D 90, 025032 (2014).

[36] J. Wang, H. Cao, J. Jing, and H. Fan, Phys. Rev. D 93, 125011 (2016).

[37] André G. S. Landulfo, and George E. A. Matsas, Phys. Rev. A 80, 032315 (2009).

[38] L. C. Céleri, André G. S. Landulfo, R. M. Serra, and George E. A. Matsas, Phys. Rev. A 81, $062130(2010)$.

[39] Z. H. Tian, J. C. Wang, and J. L. Jing, Ann. Phys. (N.Y.) 332, 98 (2012).

[40] J. C. Wang, Z. H. Tian, J. L. Jing, and H. Fan, Phys. Rev. A 93, 062105 (2016).

[41] D. E. Bruschi, J. Louko, E. Martín-Martínez, A. Dragan, and I. Fuentes, Phys. Rev. A 82, $042332(2010)$.

[42] Shun long Luo, Phys. Rev. A 77, 042303 (2008).

[43] Q. Y. He and M. D. Reid, Phys. Rev. A 88, 052121 (2013).

[44] S. Kiesewetter, Q. Y. He, P. D. Drummond, and M. D. Reid, Phys. Rev. A 90, 043805 (2014).

[45] Q. Y. He, L. Rosales-Zrate, G. Adesso, and M. D. Reid, Phys. Rev. Lett. 115, 180502 (2015). 